\title{
Exosomes in the tumor microenvironment of cholangiocarcinoma: current status and future perspectives
}

\author{
Kai Zhao ${ }^{1}$, Xiangyu Li ${ }^{1}$, Yuanxin Shi ${ }^{1}$, Yun Lu' ${ }^{1}$, Peng Qiu' ${ }^{1}$, Zhengdong Deng ${ }^{1}$, Wei Yao ${ }^{2^{*}}$ and Jianming Wang ${ }^{1,3^{*}}$
}

\begin{abstract}
Cholangiocarcinoma (CCA) refers to an aggressive malignancy with a high fatality rate and poor prognosis. Globally, the morbidity of CCA is increasing for the past few decades, which has progressed into a disease that gravely endangers human health. Exosomes belong to a class of extracellular vesicles (EVs) with diameters ranging from 40 to $150 \mathrm{~nm}$ that can be discharged by all living cells. As communication messengers of the intercellular network, exosomes carry a diverse range of cargoes such as proteins, nucleic acids, lipids, and metabolic substances, which are capable of conveying biological information across different cell types to mediate various physiological activities or pathological changes. Increasing studies have demonstrated that exosomes in the tumor microenvironment participate in regulating tumorigenesis and progression via multiple approaches in the tumor microenvironment. Here, we reviewed the current research progress of exosomes in the context of cancer and particularly highlighted their functions in modulating the development of CCA. Furthermore, the potential values of exosomes as diagnostic and therapeutic targets in CCA were overviewed as well.
\end{abstract}

Keywords: Exosomes, Cholangiocarcinoma, Tumor microenvironment, Liquid biopsy, Biomarker, Targeted therapy

\section{Background}

CCA remains a highly lethal malignancy of the biliary system. It can be classified into three major groups based on their lesion locations: intrahepatic, perihilar, and distal CCA. Globally, the proportion of CCA is second only to hepatocellular carcinoma ( $\mathrm{HCC}$ ) among all primary liver tumors, accounting for roughly $15 \%$, and accounts for $3 \%$ of all gastrointestinal malignant tumors [1, 2]. Despite advancements advances in CCA cognition, diagnosis, and treatment for the past few years, due to its high

\footnotetext{
*Correspondence: yw13557@163.com; wjm18jgm@aliyun.com ${ }^{1}$ Department of Biliary and Pancreatic Surgery/Cancer Research Center Affiliated Tongji Hospital, Tongji Medical College, Huazhong University of Science and Technology, Wuhan 430030, Hubei, China

${ }^{2}$ Department of Oncology Affiliated Tongji Hospital, Tongji Medical

College, Huazhong University of Science and Technology, Wuhan 430030, Hubei, China

Full list of author information is available at the end of the article
}

malignant degree, strong invasiveness, and the occult in initial, most patients are first diagnosed already in the late-stage that severely constrains therapeutic options. Although curative surgery is a preferred selection for some early-stage patients, the fact of tumor recurrence or metastasis after resection remains frustrating. Meanwhile, on account of the high heterogeneity of CCAs, the systemic therapeutics bring little effect for advanced patients who are not possible for radical surgery, leading to a very poor prognosis that only $7 \%-20 \%$ of patients can reach the five-year survival $[3,4]$. Nevertheless, with the advancements in genetic profiling of CCAs, emerging treatments such as targeted or immunological therapeutics may be able to assist patients with this deadly cancer to get better outcomes. Considering the current situation of CCAs, exploring new diagnostic and therapeutic strategies remains a matter of priority.

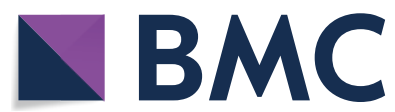

(c) The Author(s) 2022. Open Access This article is licensed under a Creative Commons Attribution 4.0 International License, which permits use, sharing, adaptation, distribution and reproduction in any medium or format, as long as you give appropriate credit to the original author(s) and the source, provide a link to the Creative Commons licence, and indicate if changes were made. The images or other third party material in this article are included in the article's Creative Commons licence, unless indicated otherwise in a credit line to the material. If material is not included in the article's Creative Commons licence and your intended use is not permitted by statutory regulation or exceeds the permitted use, you will need to obtain permission directly from the copyright holder. To view a copy of this licence, visit http://creativecommons.org/licenses/by/4.0/. The Creative Commons Public Domain Dedication waiver (http://creativeco mmons.org/publicdomain/zero/1.0/) applies to the data made available in this article, unless otherwise stated in a credit line to the data. 
EVs are enveloped by a lipid bilayer that can be discharged by numerous cell sorts. According to the size and formation pathway of vesicles, it can be classified into two major subsets approximately, called ectosomes and exosomes [5]. The former are vesicles with a diameter of $50 \mathrm{~nm} \sim 1 \mu \mathrm{m}$ via plasma membrane budding outward directly, while exosomes are EVs ranging from $40 \sim 150 \mathrm{~nm}$ in diameter generated in the opposite way, which involves plasma membrane invagination and endosomal formation [5]. Exosomes contain multiple substances and are broadly distributed in different body fluids like plasma, urine, bile, and cerebrospinal fluid (CSF), which play important roles in a variety of normal or abnormal biological behaviors [6-8]. Recently, researches about cancer exosomes have received tremendous attention. Intercellular communication in the microenvironment plays a significant role in regulating tumor development, where exosomes are key messengers that mediate this cell-to-cell communication [5, 9]. Previous researches have illustrated that exosomes participate in tumorigenesis or metastasis in multiple ways, their potential usages in cancer diagnosis and prognosis have also been deeply explored [9]. Although certain studies have reviewed the roles of EVs in the progression of CCA [10], a more comprehensive summarization of exosomes in CCA remains insufficient up to now.

In this article, we systematically summarized the research status of exosomes in the tumor fields. Based on the existing researches of exosomes in CCA, we specifically emphasized their significant roles in regulating tumor development and potential values in diagnosis and treatment.

\section{Research status of exosomes}

\section{Biogenesis, secretion and internalization}

As a type of EVs, the synthesis progress of exosomes involves three major phases: 1) plasma membrane invagination and early endosomes formation. 2) intraluminal vesicles (ILVs) and intracellular multivesicular bodies (MVBs) generation. 3) the fusion of MVBs and plasma membrane leads to exosomes secretion [9]. Generally, the biogenesis of MVBs mainly depends on the following two pathways: endosomal sorting complexes required for transport (ESCRT)-dependent or ESCRT-independent mechanisms, and the former is the most classic pathway [6]. Once mature, MVBs can integrate with autophagosomes then degrade through the lysosomal pathway or secrete into extracellular space as exosomes by fusing with the plasma membrane [11]. In this biogenesis and secretion process, other components such as tumor susceptibility gene 101 (TSG101), Rab family of GTPases (like Rab27A and Rab27B), soluble N-ethylmaleimidesensitive factor attachment protein receptor (SNARE) complexes, apoptosis-linked gene 2-interacting protein $\mathrm{X}$ (Alix), ceramide, tetraspanins (CD63, CD9, CD81), and phospholipids are also getting involved $[5,12,13]$.

Considering the difference in the origin and microenvironment, exosomes have a strong heterogeneity, which is mainly reflected in the regulation of the target cell functions [5]. Once exosomes are secreted by the host cells, they can be absorbed by target cells through various approaches like endocytosis, plasma membrane integration, and specific protein interactions [14]. Among these internalization ways, endocytosis is the most widely studied pattern. According to the characteristics of components involved in endocytosis, several subtypes are broadly divided up, including phagocytosis, macropinocytosis, clathrin-mediated endocytosis (CME), and caveolin-dependent endocytosis (CDE), as well as lipid raft-mediated internalization [15]. Moreover, several proteins such as tetraspanins, integrins, proteoglycans, and lectins, also participate in the internalization of exosomes by the unique ligand-receptor interactions[9]. However, on account of the heterogeneity of exosomes, whether or not exosomes uptake is specific remains controversial(15). Therefore, it is essential to further investigate the detailed routes of exosomes uptake (Fig. 1).

\section{Isolation and identification}

Currently, frequently-used isolation strategies include centrifugation (differential or density gradient centrifugation), particle size separation, size-exclusion chromatography, microfluidic technique, and immunoaffinity capture [16]. Until now, the most common method is still differential centrifugation due to its high exosome yields and relatively cheap price. However, it also has some deficiencies like complicated procedures, low separation efficiency, and susceptibility to contamination by soluble substances in cell culture medium or other body fluids [17]. Other isolation methods like size-exclusion chromatography, with a relatively high yield but difficult to achieve mass production, and immunoaffinity capture, advanced in specific separation yet costly with low yields $[16,17]$. So far there is not a standardized method that can achieve both economic and high purity at the same time. Therefore, the exploration of better purification methods remains a major challenge in the exosomerelated fields.

In terms of identification, the International Society of Extracellular Vesicles proposed to identify exosomes mainly from the following three aspects: 1) Exosomal morphology identification, 2) Exosomal size detection, 3) Exosomal biomarkers identification [18, 19]. Among them, transmission electron microscopy (TEM), cryo-electron microscopy (Cryo-EM), and atomic force microscopy (AFM) are the most direct methods for visual 


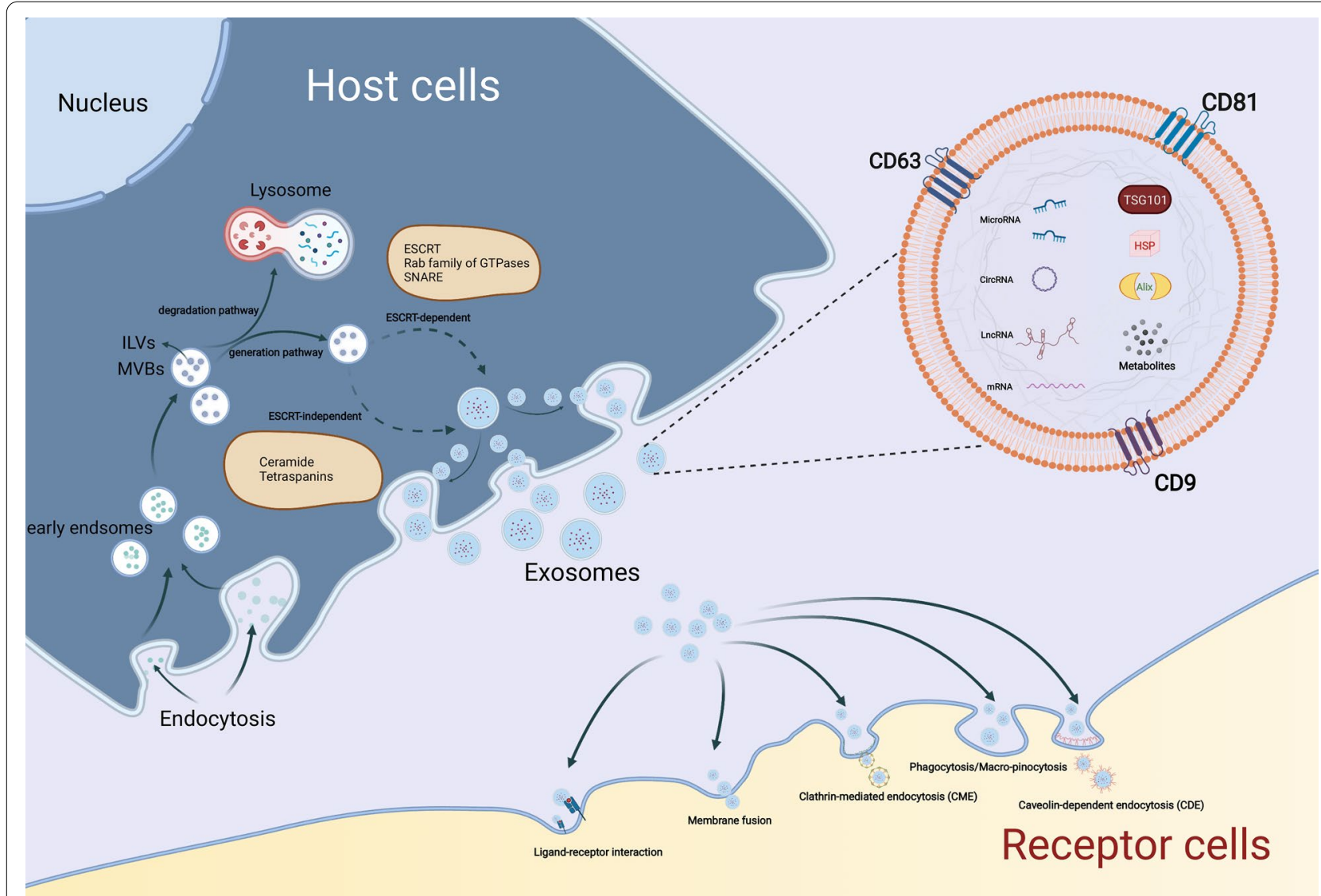

Fig. 1 Biogenesis, secretion, and internalization of exosomes. The formation of exosomes initially depends on the invagination of the plasma membrane, followed by the generation of ILVs and MVBs. Once mature, MVBs can fuse with lysosomes and be degraded, or integrate with the plasma membrane and finally get released, i.e., exosomes. During this process of synthesis and secretion, ESCRT-dependent and ESCRT-independent mechanisms are two common approaches, other components like the Rab family of GTPases, SNARE, ceramide, and tetraspanins are also involved. Exosomes can be uptake by receptor cells to perform specific functions through various mechanisms, such as phagocytosis, macro-pinocytosis, ligand-receptor interaction, CME, and CDE. As plasma membrane-derived vesicles with lipid bilayer structure, exosomes carry a variety of components, including RNAs (mRNA, MiRNA, LnCRNA, and CircRNA), proteins (TSG101, Alix, HSP, CD9, CD63, and CD81) and metabolites, etc.

observation of exosomes [20]. Real-time nanoparticle tracking technology based on the principle of Brownian motion can be used to obtain the size distribution of exosomes [21]. In addition, enzyme-linked immunosorbent assay (ELISA), flow cytometry (FCM), and western blotting (WB) are available means to detect the specific proteins or other markers expressed on exosomes [20, 22]. Reportedly, several transmembrane proteins like CD9, CD63, and CD81 are considered to be representative hallmarks, however, a recent study suggested that compared with other tetraspanins, CD63 is the unique biomarker, while CD9 and CD81 are not specific for exosomes [23]. Moreover, other components related to the formation of exosomes such as Alix, TSG101, and
Heat shock proteins (HSP) can also serve as classical hallmarks [5].

\section{The roles of exosomes in malignancies}

Since exosomes have played an essential role in multiple pathological changes through mediating intercellular communication, it has also received enormous concerns in the cancer area over the past few years [9]. Related studies have pointed out that cancer-cell-derived exosomes can modulate tumor progression through a variety of mechanisms $[9,24]$. Besides, as mentioned above, exosomes contain complex cargoes that are widespread in various body fluids, which also partly represent the heterogeneity of their parental cells, making them available for cancer diagnosis and prognostic by serving 
as novel biomarkers [25]. Moreover, recent studies have focused more on the tumor microenvironment (TME), where the signal interaction mediated by exosomes also makes a difference in tumor development $[5,26]$.

Exosomes induce or accelerate tumorigenesis. Exosomes secreted by HCC cancer cells promoted tumorigenesis through the Hedgehog pathway [27]. Mirna224-5p-enriched exosomes secreted by non-small cell lung cancer (NSCLC) cells accelerated neoplasia by directly binding with the androgen receptor (AR) [28]. On the contrary, exosomes distributed in the plasma of patients with medulloblastoma inhibited tumorigenesis by targeting FOXP4 (forkhead box protein 4) and EZH2 (enhancer of zeste 2 polycomb repressive complex 2 subunit) directly through their miRNA cargoes [29].

Exosomes have also been shown to be involved in tumor angiogenesis, which is a critical step in tumor progression. Exosomes loaded with miR-205 secreted by tumor cells induced angiogenesis via the PTEN(phosphatase and tensin homolog)/AKT pathway in Ovarian cancer [30]. Exosomal miR-25-3p derived from Colorectal cancer (CRC) cells could be absorbed by endothelial cells to facilitate, angiogenesis and increasing vascular permeability via targeting KLF2 (kruppel like factor 2) and KLF4 (kruppel like factor 4). Moreover, both in vitro study and clinical data suggested that exosomal miR-25-3p also related to the formation of pre-metastatic niche, making it a promising sign for CRC metastasis [31]. In the context of cancer, soluble E-cadherin (sE-cad)-enriched exosomes were potent stimulators of angiogenesis and might may relate to the formation of malignant ascites and widespread peritoneal metastasis in ovarian cancer patients [32].

TME consists of a group of cellular and noncellular components, including fibroblasts, immune cells such as macrophages, neutrophils, and lymphocytes, as well as cytokines, blood vessels, and extracellular matrix, etc [26]. And the crosstalk among different cell types mediated by exosomes has proven to be strongly related to tumor progression and therapeutic response. For one perspective, cancer-cell-derived exosomes regulate the function of stromal cells in the microenvironment. For example, exosomes from HCC cells induced the activation of cancer-associated fibroblasts (CAFs) activation to promote lung metastasis through their miRNA cargoes [33]. Exosomes secreted by epithelial ovarian cancer (EOC) cells under hypoxic conditions could regulate the macrophage polarization by transferring their miRNAs (miR-21-3p, miR-125b-5p, miR-181d-5p) to promote tumor proliferation and metastasis [34]. From another perspective, exosomes originated from other infiltrating cells in the TME can also modulate the biological behavior of cancer cells. Exosomal miR-34a-5p could transfer from CAFs to cancer cells, subsequently induced epithelial-mesenchymal transition (EMT) via the AKT/GSK$3 \beta$ (glycogen synthase kinase 3 beta) $/ \beta$-catenin pathway in oral squamous cell carcinoma (OSCC) [35]. Exosomes derived from M2 macrophage mediated an intercellular transfer of the integrin $\alpha_{M} \beta_{2}$ and promoted HCC metastasis through activating the MMP9 (matrix metalloproteinase 9) signaling pathway [36]. Moreover, the functions of exosomes in tumor immunity have also been explored to some degree. Hypoxia-induced tumor exosomes were abundant in chemokines and cytokines like CSF-1(colony stimulating factor 1), MCP-1(monocyte chemoattractant protein-1), and TGF $\beta$ (transforming growth factor beta), which can modify the host immune microenvironment and enhance tumor progression via influencing the macrophage recruitment and polarization [37]. Circ-UHRF1 (ubiquitin-like with PHD and ring finger domain 1), existed in plasma exosomes secreted by HCC patients, which leading to immunosuppression by inhibiting NKs (natural killer cells) activity via circUHRF1/miR-449c-5p/ TIM-3( $\mathrm{T}$ cell immunoglobulin domain and mucin domain 3) axis [38]. While such exosome-mediated signal transmission can exert antitumor effects under certain circumstances. For example, exosomes derived from DCs (dendritic cells) were reported that might become a novel vaccine applied in tumor immunotherapy. Exosomes secreted by $\alpha$-fetoprotein (AFP)-positive DCs could effectively improve the immune microenvironment of mice models with HCC, making it a hopeful new strategy for immunotherapy of HCC [39]. In addition to the components contained in exosomes, their external molecules also participate in tumor immunoregulation. PD-L1(programmed death 1 ligand), known as a natural ligand for PD-1 (programmed death 1), can suppress the immunocompetence of $\mathrm{T}$ cells, $\mathrm{B}$ cells, and monocytes by directly binding with PD-1 on their surface to promote tumor immune escape [40]. It has been demonstrated that anti-PD-1/PD-L1 therapeutics has achieved a great success in multiple cancers, including metastatic melanoma, NSCLC, glioblastoma, and colon cancer, while the problem of drug resistance largely limits their clinical application [41]. Recent researches have indicated that exosomes derived from cancer cells also express PD-L1. PD-L1 ${ }^{+}$exosomes can impair immune functions and promote tumor growth in the similar way as above, which may also result in a low response to anti-PD-L1 therapy. Therefore, targeting the PD-L1 expressed on exosomes is expected to improve the present situation of cancer immunotherapy [40, 42].

Palliative treatments like radiotherapy, chemotherapy, and targeted therapy are recommended choices for the later period tumor patients, while it is also disappointing because tumors are prone to become tolerant of these 
chemotherapeutic agents or antibodies. And increasing evidence supports that exosomes can facilitate this resistance by mediating intercellular communication. LncRNA SNHG7 (small nucleolar RNA host gene 7) transmitted between cancer cells in exosomes enhanced docetaxel resistance in lung adenocarcinoma (LUAD) by inducing autophagy and regulating the macrophage polarization [43]. And tumor-associated macrophages (TAMs) derived exosomes were capable of inducing drug resistance in multiple cancers through their miRNA or IncRNA cargoes as well [44-47]. Moreover, miRNA522, abundant in exosomes released from CAFs, could promote acquired chemotherapy resistance and inhibit ferroptosis, thus supporting tumor progression of gastric cancer [48]. In addition, other researchers have also noted that exosomes play a unique role in improving drug resistance. For example, miR-567-enriched exosomes reversed trastuzumab resistance via suppressing autophagy by targeting ATG5(autophagy related 5), promising to serve as a potential therapeutic target for breast cancer patients [49].

As for the value of exosomes in tumor diagnosis and prognosis, there is a lot of researches to back it up. Previous studies mainly focused on its nucleic acids cargoes, especially small RNA molecules such as classical oncogenic miR-21, miR-155, and anti-oncogenic miRNAs like miR-146a and miR-34a, which are differentially expressed between tumor cells and non-tumor cells, enabling them to diagnose multiple cancers at an early-stage, like pancreas, colorectum, liver, and breast cancers [50]. Besides miRNAs, exosomal proteins also have clinical significance [51]. Glypican-1 (GPC1)-positive exosomes could be used as an early diagnosis tool for patients with pancreatic cancers, which also performed better in prognosis prediction compared with CA19-9 [52]. By constructing mouse liver damage models and the proteomic analysis of their urine exosomes, twenty-eight novel proteins were identified and four of them are promising were expected to be used as non-invasive indicators in hepatic disease [53]. Based on the evidence of these researches, the combination of multiple components involved in exosomes may help enhance the specificity and sensitivity of cancer diagnosis, while further research is still needed.

To sum up, exosomes are closely related to tumor progression, and it has been well explored in a wide range of digestive malignancies. However, relevant studies in CCA are still insufficient, and existing researches have not been systematically described either. Here, the current research progress of exosomes in CCA will be comprehensively introduced in the next part.

\section{Roles of exosomes in CCA}

\section{Exosomes in CCA progression}

As the most well-studied component of exosomes, the critical roles of ncRNAs in CCA have been extensively reported as well. Several dysregulated exosomal miRNAs have been detected between CCA cells and normal biliary epithelial cells through miRNA profiling analysis. Among these differentially expressed miRNAs, miR-205-5p, miR-200c-3p and miR-200b-3p, were significantly abundant in exosomes, while miR-34c-5p and miR-199 clusters were down-regulated distinctly. Subsequently, KEGG (kyoto encyclopedia of genes and genomes) enrichment analysis of target genes predicted by differentially expressed miRNAs suggested that dysregulated miRNAs were closely related to multiple cancer-associated pathways, and down-regulating the expression of miR-205-5p effectively suppressed the invasion and metastasis of CCA cells in vitro, indicating that it might take an essential part in CCA progression [54]. And in the CCA microenvironment, EVs derived from cancer cells could induce bone marrow mesenchymal stem cells (BMSCs) to differentiate into fibroblasts, accompanied by a significant up-regulation in the myofibroblast markers like $\alpha$-SMA (alpha-smooth muscle actin), FAP (fibroblast activation protein alpha), and vimentin. Such differentiation enhanced the migratory capability of BMSCs and contributed to tumor extracellular matrix formation, facilitating the development of CCA ultimately. As a response, some soluble mediators such as IL-6 (interleukin- 6) could be selectively released from BMSCs as well, and in turn, enhancing the proliferation capacity of tumor cells by stimulating the STAT3 (signal transducer and activator of transcription 3) signaling pathway [55]. Another study focused on the interaction between CCA cells and fibroblasts observed that down-regulating the miR$34 \mathrm{c}$ in exosomes derived from CCA cells could mediate the activation of CAFs to promote cancer development [56].

Interestingly, certain miRNAs transferred in the form of exosomes can also act as tumor suppressors. In the co-culture system of CCA cell line HuCCT1 and hepatic stellate cell line LX2, several markedly down-regulated miRNAs in LX2 cells were identified, and among them, miR-195 received more attention. Further functional experiments showed that the capacities of growth and invasion of CCA cells decreased significantly after upregulating the miR-195 in LX2 cells, indicating that miR195 might act as a tumor suppressor in this cell culture model. Subsequent studies on the mechanism demonstrated that miR-195 was loaded in EVs secreted by LX2 cells, and performed this anti-tumor effect by directly transferring to CCA cells [57]. Similarly, intercellular 
transmission of miR-30e in EVs can also inhibit EMT of receptor cells via targeting Snail and then suppress invasion and metastasis of CCA [58].

Besides miRNAs, other exosomal ncRNAs like circRNA and lncRNA have been reported in CCA as well. Circ-0000284 was markedly up-regulated in CCA cells compared to normal bile duct cells, promoted CCA development as a competitive endogenous RNA via miR-637/LY6E (lymphocyte antigen 6 family member E) pathway. The study also pointed out that circ-0000284 transmission via exosomes could induce a malignant transformation of normal cells adjacent to cancer [59]. Circ-CCAC1 (Cholangiocarcinoma-associated circular RNA 1) facilitated CCA growth and migration via sponging miR-514a-5p to elevate the expression of YY1 (Yin Yang 1) and CAMLG (calcium modulating ligand). And a high level of circ-CCAC1 was detected CCA-derived EVs, these EVs could be transmitted to endothelial cells to disrupt the continuity of vascular endothelial barrier and stimulate the formation of new blood vessels, promoting tumor growth and metastasis in turn [60].

In addition to nucleic acid molecules, the protein components contained in exosomes are also related to the malignant progression of CCA. For example, FZD 10 (frizzled class receptor 10) proteins, related to the Wnt signaling pathway, were detected in CCA-derived exosomes that could promote cell proliferation and might be involved in mediating the cancer reactivation and distant metastasis [61]. Several common cancer-related proteins, like integrin $\alpha / \beta$, lactadherin, and vitronectin, were identified in CCA-derived exosomes, could induce invasion and migration of cholangiocytes by up-regulating the expression of $\beta$-catenin [62].

As mentioned above, exosomes also participate in regulating tumor progression by modulating the immune microenvironment. And in CCA, Chen et al. have observed that cancer-related exosomes could reduce the population of cytokine-induced killer cells (CIKs), leading to a secretory reduction of TNF- $\alpha$ (tumor necrosis factor- $\alpha$ ) and perforin, thus inhibiting anti-tumor activity and promoting tumor immune escape ultimately [63].

In conclusion, these studies have confirmed that exosomes are closely related to the progression of CCA, providing a new perspective for understanding the regulatory mechanisms of tumor development (Fig. 2).

\section{Exosomes in CCA diagnosis}

Due to the stealthiness and heterogeneity of CCA, traditional image logical examination or laboratory test cannot achieve early diagnosis or dig out characteristics that reflect tumorigenesis. Recently, an emerging method of diagnosis called "liquid biopsy" has attracted more attention that may make up for the deficiency of traditional detection methods in cancer diagnosis. Several explorations aimed to develop the exosomes as objects for liquid biopsy have also been conducted in CCA.

Several differentially expressed exosomal RNAs have been detected in serum or urine of CCA patients, compared with primary sclerosing cholangitis (PSC), ulcerative colitis (UC), and healthy controls. Among them, five serum exosomal mRNAs including CMIP (c-Maf inducing protein), GAD1 (glutamate decarboxylase 1), NDKP1 (nucleoside diphosphate kinase 1), CDS1 (CDPdiacylglycerol synthase 1), and CKS1B (cyclin-dependent kinase regulatory subunit 1 ), as well as a cluster of urine exosomal mRNAs consisted of UBE2C (ubiquitin-conjugating enzyme E2C) and SERPINB1 (serine protease inhibitor B1), showed an excellent performance in diagnostic efficiency. Particularly, uniting CMIP, NDKP1, and CKS1B performed a better diagnostic power. Moreover, exosomal ncRNAs [miR-604, miR-551b, and lncRNA MALAT1(metastasis associated lung adenocarcinoma transcript 1)] in the serum of CCA patients also contributed to the diagnosis [64]. Similarly, in another study of serum exosomes, twenty exosomal miRNAs were identified and five of them had a significant differential expression. Further assessment of diagnostic value using these dysregulated miRNAs showed that miR-200 family members (miR-141-3p, miR-200a-3p, miR-200b-3p, and miR200c-3p) performed better than CA19-9. Particularly miR-200c-3p, which is positively related to tumor stage, was expected to be a novel serum indicator for CCA progression [65]. Conversely, certain miRNAs downregulated in exosomes also possessed diagnostic values, such as miR-214-3p and miR-199 family members [54]. And the latest research noted that Cripto-1 [also named teratocarcinoma-derived growth factor 1 (TDGF-1)], largely existed in exosomes isolated from the serum of perihilar cholangiocarcinoma (PHCCA) patients versus cholangitis patients and normal controls, performed a better diagnostic capacity than CEA and CA19-9 (AUC: 0.82; Sensitivity: 79.1\%; Specificity: $87.5 \%$ ). Furthermore, Immunohistochemistry analysis using PHCCA tissues showed a negative correlation between Cripto-1 and E-cadherin, indicating that Cripto-1 was also related to EMT and may possess an independent prognostic significance in PHCCA [66].

Researches aimed at bile-derived exosomes have also identified several circulating exosomal lncRNAs, which are closely related to oncogenic signaling pathways (like p53 and RAS signaling pathways) that may be conducive to the diagnosis of CCA [67]. Another high-quality research has identified four miRNAs (miR-96-5p, miR191-5p, miR-151a-5p, and miR-4732-3p) that enriched in exosomes isolated from blood samples of CCA patients, 


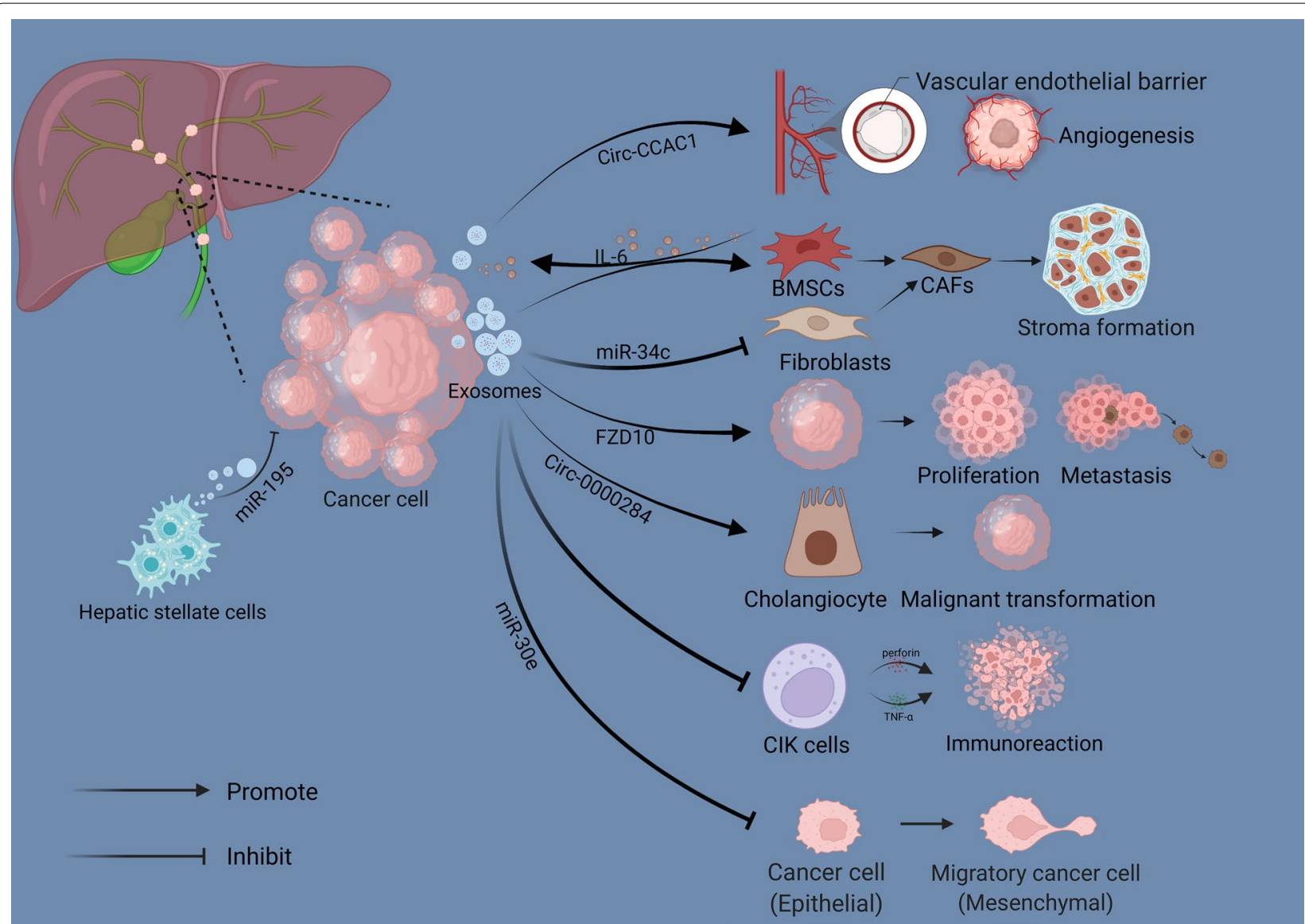

Fig. 2 The functional network of exosomes in the CCA microenvironment. Exosomes secreted by cancer cells facilitate tumor angiogenesis by transferring Circ-CCAC1 to endothelial cells. Cancer cell-derived exosomes induce BMSCs to differentiate into CAFs to promote tumor stroma formation, and normal fibroblasts can also be activated through their miRNA cargoes, like miR-34c. Other RNAs cargoes, such as Circ-0000284 can promote tumor development by inducing a malignant transformation of normal cholangiocytes, while miR-30e-enriched exosomes inhibit tumor progression via suppressing EMT. Similarly, exosomes derived from Hepatic stellate cells exert an anti-tumor effect by transferring miR-195. Certain protein components in exosomes can also promote tumor proliferation and metastasis, like FZD10. CIKs absorb exosomes to promote tumor immune escape by reducing the secretion of TNF- $a$ and perforin

which are promising for early diagnosis of CCA, especially stage II patients [68].

In addition, some researchers have paid more attention to the proteins contained in CCA exosomes. By using proteomics approaches, Heat shock protein 90 (HSP90) was confirmed that differentially phosphorylated in invasive CCA cells and was expected to serve as an implement for indicating the metastatic of CCA [69]. Besides, proteomic analysis showed that Claudin-3 was enriched in human bile-derived exosomes, and might become a novel biomarker for CCA as well [70]. In comparison with serum exosomes isolated from normal individuals, several specific proteins were observed to be concentrated in exosomes of CCA patients. In detail, a combination of three of them, including AMPN (aminopeptidase $\mathrm{N})$, VNN1 (pantetheinase), and PIGR (polymeric immunoglobulin receptor), performed better in diagnosis that might become alternative serum biomarkers in CCA. And in the same study, another three exosomal proteins: FIBG (fibrinogen gamma chain), A1AG1 (alpha1-acid glycoprotein 1), and S100A8 (S100 calcium binding protein A8), were expected to become an effective basis for differential diagnosis with PSC [71].

In summary, these researches have emphasized the potential value of exosomes in CCA diagnosis and prognosis, while large cohort validation is still needed in the future (Table 1).

\section{Progression in adjuvant therapy of CCA and the potential application of exosomes}

At present, for CCA patients at an advanced stage that cannot accept radical surgery, gemcitabine combined with cisplatin is the preferred recommended option. More than three months longer median survival time is 


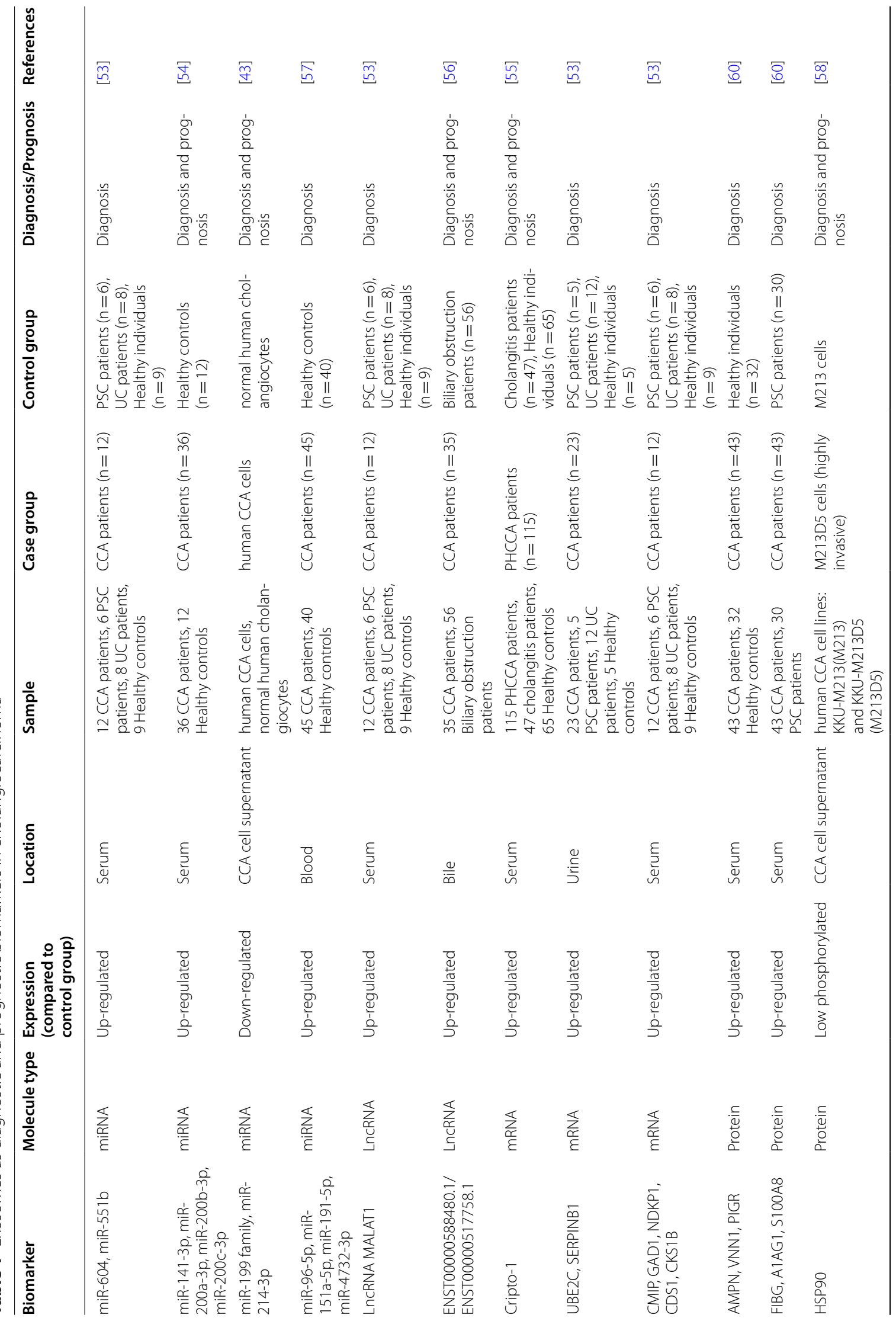




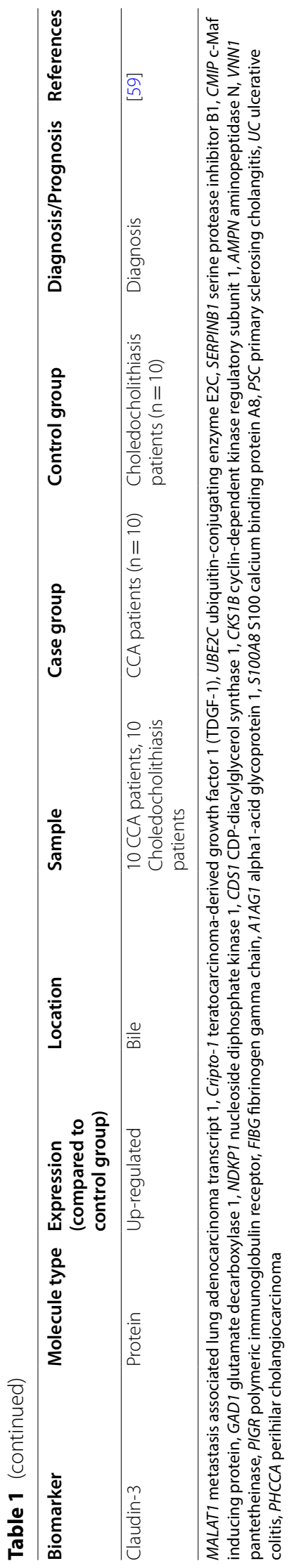


observed in patients who received this combination compared to gemcitabine treatment alone (gemcitabine + cisplatin group: 11.7 months; gemcitabine: 8.1 months) [72]. In addition, Rachna et al. have also reported that treatment with gemcitabine-cisplatin plus nab-paclitaxel displayed a better survival benefit versus gemcitabinecisplatin alone in a phase II clinical trial [73], promising to become a new first-line therapeutic regimen for CCA. However, due to the significant heterogeneity of CCA, chemotherapy-based systemic treatments often have limited efficacy [74]. In recent years, with the maturity of genomics approaches, the molecular pathological mechanism of cholangiocarcinoma has been revealed gradually, providing new possibilities for individualized and targeted therapies [75].

Studies have found that different genomic profiles are shown in three CCA subtypes. To be more specific, patients with iCCA show a high mutation frequency in IDH1/2, KRAS, BAP1, TP53, as well as FGFR fusions, while PRKACA, PRKACB, and ELF3 mutations are more likely to appear in pCCA/dCCA patients [76, 77]. According to these mutations and fusions, CCA can be further divided into different molecular subtypes and prognostic assessment becomes available on this basis. Furthermore, several targeted drugs aimed at these mutations have already entered clinical trials [75]. For example, infigratinib (NVP-BGJ398) and erdafitinib, known as pan-FGFR inhibitors, displayed an excellent anti-tumor capacity, with a positive therapeutic response and controllable security, observed from relevant phase I and phase II clinical data [78, 79]. Moreover, immunotherapeutic strategies represented by anti-PD-1/anti-PD-L1 monoclonal antibodies have shown a significant remission rate in multiple human malignancies [80]. In advanced biliary tract cancers, several clinical trials have also reported that some progress has been made in patients who received pembrolizumab therapeutic (an immune checkpoint inhibitor targeting PD-1), with an objective response rate varying from $5.8 \% \sim 13 \%$ and a median progression-free survival of about 2 months [81]. However, relevant clinical exploration is still insufficient and unsatisfactory in CCA.

Exosomes are lipid bilayer-enveloped extracellular structures that can protect their cargoes from degradation. And most importantly, exosomes secreted by different cells can partly represent the heterogeneity of their parental cells, conferring them excellent biocompatibility compared to other drug delivery vehicles like liposomes or lipid-based nanoparticles. Therefore, it seems that exosomes are more suitable as drug carriers for cancer treatment [5]. Compared to direct therapy, exosomes as drug carriers perform better uptake efficiency and lower toxicity. Engineering exosome content to transport therapeutic nucleic acids, proteins, or drugs directly to cancer cells shows an exciting potential in cancer treatment [82]. For example, miR-122, which acted as an anti-tumor miRNA in HCC, could inhibit cancer development via suppressing EMT and angiogenesis and enhancing the chemosensitivity [83]. It was reported that adipose tissue-derived mesenchymal stem cells (AMSCs) derived exosomes could effectively transfer miR-122 to HCC cells, thereby increasing HCC chemosensitivity by modulating the expression of downstream molecules [84]. Similarly, transferring miR-199a-enriched exosomes from modified AMSCs to HCC cells enhanced the sensitivity of doxorubicin treatment by inhibiting the mTOR (mechanistic target of rapamycin kinase) signaling pathway [85]. Moreover, patients with pancreatic ductal adenocarcinoma are susceptible to becoming chemoresistant, leading to a dismal therapeutic response and a poor prognosis. To improve this current status of treatment, Zhou et al. have loaded paclitaxel and gemcitabine monophosphate (an intermediate product of gemcitabine metabolism) into BMSCs-derived exosomes which can be absorbed by tumor cells. Further experiments showed an excellent penetration based on this Exo delivery platform, contributing to a favorable anti-tumor efficacy and relatively mild systemic toxicity [86]. These findings have opened up the way for exosomes to be used in tumor-targeted therapy.

Nevertheless, the application of exosomes as therapeutic targets in CCA is still in infancy, the current research focus is still inclined to their roles in tumor diagnosis and development, as we mentioned before. As for their therapeutic potential, existing studies have found that transferring several tumor-suppressive miRNAs (miR195 and miR-30e) through exosomes can effectively inhibit CCA development $[57,58]$. Vaccination of hamsters combined with Opisthorchis viverrini EVs and their surface recombinant tetraspanins has been demonstrated to reduce the burden of infection through inducing antibody reactions, exerting a protective role to avoid the appearance of CCA [87]. A relevant study reported that methotrexate-equipped EVs derived from cancer cells could effectively relieve malignant biliary obstruction by inducing pyroptotic cell death in patients with CCA [88]. Additionally, the latest research focused on the TME of CCA also brings forceful support for EV-based therapeutics. Exosomal miR-183-5p derived from cancer cells suppressed immune responses and promoted iCCA progression through up-regulating the expression of PD-L1 in macrophages via miR-183-5p/PTEN/AKT axis, promising to be a novel target for overcoming therapeutic tolerance of immune checkpoint inhibitors in iCCA [89]. Moreover, circ-0020256, produced by TAMs and loaded 
in exosomes, significantly facilitated the proliferation and metastasis of CCA cells, cutting off the cross-talk mediated by exosomal circ-0020256 between CCA cells and TAMs might be a hopefully therapeutic strategy [90]. All these findings provide strong evidence to support the application of exosomes in the treatment of CCA. However, mainly of them are based only on animal experiments, meaning that there is still a long way from clinical trials.

\section{Conclusions and perspectives}

A growing number of studies support the thesis that the microenvironment plays a significant role in the development of multiple human cancers. Given that exosomes are key components of the microenvironment and act as messengers of intercellular communication, relevant studies have become hot topics of cancer research.

Exosomes can be separated from various body fluids, and certain RNAs, proteins, or metabolites involved in them have advantages in stability and abundance, giving them unique advantages in tumor diagnosis and prognosis. Moreover, since exosomes have excellent histocompatibility and can partly reflect the heterogeneity of their parental cells, modification of exosomes for cancer treatment also shows great potential. However, there are still many problems to be solved in the clinical application of exosomes. First, current exosome isolation and purification techniques need to be further optimized to achieve better efficiency and convenience. Next, the molecular mechanisms of exosomes in CCA malignant progression remains unclear, hence the exploration of internal details remains needed to develop potential therapeutic targets. Last but not least, the artificial synthetic exosome techniques are immature at the present stage, related clinical trials are also inadequate, thus developing exosomes as drug carriers in cancer treatment still faces lots of challenges. Nevertheless, with the continuous progress of characterization, separation, purification, and modification technologies, we firmly believe that exosomes will eventually be applied in the diagnosis and treatment of CCA.

\footnotetext{
Abbreviations

CCA: Cholangiocarcinoma; EVs: Extracellular vesicles; HCC: HepatocelIular carcinoma; CSF: Cerebrospinal fluid; ILVs: Intraluminal vesicles; MVBs: Multivesicular bodies; ESCRT: Endosomal sorting complexes required for transport; TSG101: Tumor susceptibility gene 101; SNARE: Soluble N-ethylmaleimide-sensitive factor attachment protein receptor; Alix: Apoptosis-linked gene 2-interacting protein X; CME: Clathrin-mediated endocytosis; CDE: Caveolin-dependent endocytosis; TEM: Transmission electron microscopy; Cryo-EM: Cryo-electron microscopy; AFM: Atomic force microscopy; ELISA: Enzyme-linked immunosorbent assay; FCM: Flow cytometry; WB: Western blotting; HSP: Heat shock protein; MiRNA: MicroRNA; LncRNA: Long noncoding RNA; CircRNA: Circular RNA; NSCLC: Non-small cell lung cancer; AR: Androgen receptor; FOXP4: Forkhead box protein 4; EZH2: Enhancer of zeste
}

2 polycomb repressive complex 2 subunit; PTEN: Phosphatase and tensin homolog; CRC: Colorectal cancer; KLF2: Kruppel like factor 2; KLF4: Kruppel like factor 4; sE-cad: Soluble E-cadherin; TME: Tumor microenvironment; CAFs: Cancer-associated fibroblasts; EOC: Epithelial ovarian cancer; EMT: Epithelialmesenchymal transition; GSK-3ß: Glycogen synthase kinase 3 beta; OSCC: Oral squamous cell carcinoma; MMP9: Matrix metalloproteinase 9; CSF-1: Colony stimulating factor 1; MCP-1: Monocyte chemoattractant protein-1; TGF $\beta$ : Transforming growth factor beta; UHRF1: Ubiquitin-like with PHD and ring finger domain 1; NKs: Natural killer cells; TIM-3: T cell immunoglobulin domain and mucin domain 3; DCs: Dendritic cells; AFP: a-Fetoprotein; PD-L 1: Programmed death 1 ligand; PD-1: Programmed death 1; SNHG7: Small nucleolar RNA host gene 7; LUAD: Lung adenocarcinoma; TAM: Tumor-associated macrophage; ATG5: Autophagy related 5; GPC1: Glypican-1; KEGG: Kyoto encyclopedia of genes and genomes; BMSCs: Bone marrow mesenchymal stem cells; $a-S M A$ : Alpha-smooth muscle actin; FAP: Fibroblast activation protein alpha; IL-6: Interleukin- 6; STAT3: Signal transducer and activator of transcription 3; LY6E: Lymphocyte antigen 6 family member $E_{;}$CCAC1: Cholangiocarcinoma-associated circular RNA 1; YY1: Yin Yang 1; CAMLG: Calcium modulating ligand; FZD 10: Frizzled class receptor 10; CIKs: Cytokine-induced killer cells; TNF-a: Tumor necrosis factor-a; PSC: Primary sclerosing cholangitis; UC: Ulcerative colitis; CMIP: C-Maf inducing protein; GAD1: Glutamate decarboxylase 1; NDKP1: Nucleoside diphosphate kinase 1; CDS1: CDP-diacylglycerol synthase 1; CKS1B: Cyclin-dependent kinase regulatory subunit 1; UBE2C: Ubiquitin-conjugating enzyme E2C; SERPINB1: Serine protease inhibitor B1; MALAT1: Metastasis associated lung adenocarcinoma transcript 1; Cripto-1:Teratocarcinoma-derived growth factor 1 (TDGF-1); PHCCA: Perihilar cholangiocarcinoma; AMPN: Aminopeptidase N; VNN1: Pantetheinase; PIGR: Polymeric immunoglobulin receptor; FIBG: Fibrinogen gamma chain; A1AG1: Alpha1-acid glycoprotein 1; S100A8: S100 calcium binding protein A8; iCCA: Intrahepatic cholangiocarcinoma; pCCA: Perihilar cholangiocarcinoma; dCCA: Distal cholangiocarcinoma; IDH1/2: Isocitrate dehydrogenase1/2; KRAS: Kirsten ratsarcoma viral oncogene homolog; BAP1: BRCA1 associated protein 1; TP53: Tumor protein p53; FGFR: Fibroblast growth factor receptor; PRKACA: Protein kinase CAMP-activated catalytic subunit alpha; PRKACB: Protein kinase CAMP-activated catalytic subunit beta; ELF3: E74 like ETS transcription factor 3; AMSCs: Adipose tissue-derived mesenchymal stem cells; mTOR: Mechanistic target of rapamycin kinase.

\section{Acknowledgements}

Thanks for the support from National Natural Science Foundation of China (No.81874062, 82072730), and Youth Program of National Natural Science Foundation of China (81902439). And thanks for two correspondence authors' wholehearted guidance and other author's cooperation.

\section{Authors' contributions}

KZ and WY designed the review. KZ drafted the manuscript. WY and J-MW participated in the critical revision of the manuscript. X-YL, Y-XS, YL, PQ, Z-DD participated in the search for the articles cited. All authors read and approved the final manuscript.

\section{Funding}

The work was supported by National Natural Science Foundation of China (No. 81874062, 82072730), and Youth Program of National Natural Science Foundation of China (81902439).

\section{Availability of data and materials}

Not applicable.

\section{Declarations}

Ethics approval and consent to participate Not applicable.

\section{Consent for publication}

Not applicable.

\section{Competing interests}

The authors declare no conflict of interest. 


\section{Author details}

'Department of Biliary and Pancreatic Surgery/Cancer Research Center Affiliated Tongji Hospital, Tongji Medical College, Huazhong University of Science and Technology, Wuhan 430030, Hubei, China. ${ }^{2}$ Department of Oncology Affiliated Tongji Hospital, Tongji Medical College, Huazhong University of Science and Technology, Wuhan 430030, Hubei, China. ${ }^{3}$ Affiliated Tianyou Hospital, Wuhan University of Science and Technology, Wuhan 430064, China.

Received: 2 January 2022 Accepted: 2 February 2022

Published online: 07 March 2022

\section{References}

1. Banales JM, Cardinale V, Carpino G, Marzioni M, Andersen JB, Invernizzi P, et al. Expert consensus document: Cholangiocarcinoma: current knowledge and future perspectives consensus statement from the European Network for the Study of Cholangiocarcinoma (ENS-CCA). Nat Rev Gastroenterol Hepatol. 2016;13(5):261-80.

2. Banales JM, Marin JJG, Lamarca A, Rodrigues PM, Khan SA, Roberts LR, et al. Cholangiocarcinoma 2020: the next horizon in mechanisms and management. Nat Rev Gastroenterol Hepatol. 2020;17(9):557-88.

3. Cambridge WA, Fairfield C, Powell JJ, Harrison EM, Soreide K, Wigmore SJ, et al. Meta-analysis and meta-regression of survival after liver transplantation for unresectable perihilar cholangiocarcinoma. Ann Surg 2021;273(2):240-50.

4. Bertuccio P, Malvezzi M, Carioli G, Hashim D, Boffetta P, El-Serag HB, et al. Global trends in mortality from intrahepatic and extrahepatic cholangiocarcinoma. J Hepatol. 2019;71(1):104-14.

5. Kalluri R, LeBleu VS. The biology, function, and biomedical applications of exosomes. Science. 2020;367(6478).

6. Guay C, Regazzi R. Exosomes as new players in metabolic organ crosstalk. Diabetes Obes Metab. 2017;19(Suppl 1):137-46.

7. BudnikV, Ruiz-Canada C, Wendler F. Extracellular vesicles round off communication in the nervous system. Nat Rev Neurosci. 2016;17(3):160-72.

8. Robbins PD, Morelli AE. Regulation of immune responses by extracellular vesicles. Nat Rev Immunol. 2014;14(3):195-208.

9. Liu J, Ren L, Li S, Li W, Zheng X, Yang Y, et al. The biology, function, and applications of exosomes in cancer. Acta Pharm Sin B. 2021;11(9):2783-97.

10. Bai M, Fu W, Su G, Cao J, Gao L, Huang C, et al. The role of extracellular vesicles in cholangiocarcinoma. Cancer Cell Int. 2020;20(1):435.

11. van Niel G, D'Angelo G, Raposo G. Shedding light on the cell biology of extracellular vesicles. Nat Rev Mol Cell Biol. 2018;19(4):213-28.

12. Mashouri L, Yousefi H, Aref AR, Ahadi AM, Molaei F, Alahari SK. Exosomes: composition, biogenesis, and mechanisms in cancer metastasis and drug resistance. Mol Cancer. 2019;18(1):75.

13. Mathieu M, Martin-Jaular L, Lavieu G, Thery C. Specificities of secretion and uptake of exosomes and other extracellular vesicles for cell-to-cell communication. Nat Cell Biol. 2019;21(1):9-17.

14. Gurung S, Perocheau D, Touramanidou L, Baruteau J. The exosome journey: from biogenesis to uptake and intracellular signalling. Cell Commun Signal. 2021;19(1):47.

15. Mulcahy LA, Pink RC, Carter DR. Routes and mechanisms of extracellular vesicle uptake. J Extracell Vesicles. 2014;3.

16. Li P, Kaslan M, Lee SH, Yao J, Gao Z. Progress in Exosome Isolation Techniques. Theranostics. 2017:7(3):789-804.

17. Zhu L, Sun HT, Wang S, Huang SL, Zheng Y, Wang CQ, et al. Isolation and characterization of exosomes for cancer research. J Hematol Oncol. 2020;13(1):152.

18. Witwer KW, Soekmadji C, Hill AF, Wauben MH, Buzas El, Di Vizio D, et al. Updating the MISEV minimal requirements for extracellular vesicle studies: building bridges to reproducibility. J Extracell Vesicles. 2017;6(1):1396823.

19. Lotvall J, Hill AF, Hochberg F, Buzas El, Di Vizio D, Gardiner C, et al. Minimal experimental requirements for definition of extracellular vesicles and their functions: a position statement from the International Society for Extracellular Vesicles. J Extracell Vesicles. 2014;3:26913.

20. Szatanek R, Baj-Krzyworzeka M, Zimoch J, Lekka M, Siedlar M, Baran J. The Methods of Choice for Extracellular Vesicles (EVs) Characterization. Int J Mol Sci. 2017;18(6)
21. Saveyn H, De Baets B, Thas O, Hole P, Smith J, Van der Meeren P. Accurate particle size distribution determination by nanoparticle tracking analysis based on 2-D Brownian dynamics simulation. J Colloid Interface Sci. 2010;352(2):593-600.

22. Chiriaco MS, Bianco M, Nigro A, Primiceri E, Ferrara F, Romano A, et al. Lab-on-Chip for Exosomes and Microvesicles Detection and Characterization. Sensors (Basel). 2018;18(10).

23. Mathieu M, Nevo N, Jouve M, Valenzuela JI, Maurin M, Verweij FJ, et al. Specificities of exosome versus small ectosome secretion revealed by live intracellular tracking of CD63 and CD9. Nat Commun. 2021;12(1):4389.

24. Chen $\mathrm{H}$, Chengalvala $\mathrm{V}, \mathrm{Hu} \mathrm{H}$, Sun D. Tumor-derived exosomes: Nanovesicles made by cancer cells to promote cancer metastasis. Acta Pharm Sin B. 2021;11(8):2136-49.

25. Zhou B, Xu K, Zheng X, Chen T, Wang J, Song Y, et al. Application of exosomes as liquid biopsy in clinical diagnosis. Signal Transduct Target Ther. 2020;5(1):144

26. Dai J, Su Y, Zhong S, Cong L, Liu B, Yang J, et al. Exosomes: key players in cancer and potential therapeutic strategy. Signal Transduct Target Ther. 2020;5(1):145

27. Li L, Zhao J, Zhang Q, Tao Y, Shen C, Li R, et al. Cancer Cell-Derived Exosomes Promote HCC Tumorigenesis Through Hedgehog Pathway. Front Oncol. 2021;11:756205.

28. Zhou J, Wang H, Sun Q, Liu X, Wu Z, Wang X, et al. miR-224-5p-enriched exosomes promote tumorigenesis by directly targeting androgen receptor in non-small cell lung cancer. Mol Ther Nucleic Acids. 2021;23:1217-28.

29. Xue P, Huang S, Han X, Zhang C, Yang L, Xiao W, et al. Exosomal miR101-3p and miR-423-5p inhibit medulloblastoma tumorigenesis through targeting FOXP4 and EZH2. Cell Death Differ. 2021.

30. He L, Zhu W, Chen Q, Yuan Y, Wang Y, Wang J, et al. Ovarian cancer cellsecreted exosomal miR-205 promotes metastasis by inducing angiogenesis. Theranostics. 2019;9(26):8206-20.

31. Zeng Z, Li Y, Pan Y, Lan X, Song F, Sun J, et al. Cancer-derived exosomal miR-25-3p promotes pre-metastatic niche formation by inducing vascular permeability and angiogenesis. Nat Commun. 2018;9(1):5395.

32. Tang MKS, Yue PYK, Ip PP, Huang RL, Lai HC, Cheung ANY, et al. Soluble E-cadherin promotes tumor angiogenesis and localizes to exosome surface. Nat Commun. 2018:9(1):2270.

33. Fang T, Lv H, Lv G, Li T, Wang C, Han Q, et al. Tumor-derived exosomal miR-1247-3p induces cancer-associated fibroblast activation to foster lung metastasis of liver cancer. Nat Commun. 2018;9(1):191.

34. Chen $X$, Zhou J, Li X, Wang X, Lin Y, Wang X. Exosomes derived from hypoxic epithelial ovarian cancer cells deliver microRNAs to macrophages and elicit a tumor-promoted phenotype. Cancer Lett. 2018:435:80-91.

35. Li YY, Tao YW, Gao S, Li P, Zheng JM, Zhang SE, et al. Cancer-associated fibroblasts contribute to oral cancer cells proliferation and metastasis via exosome-mediated paracrine miR-34a-5p. EBioMedicine. 2018;36:209-20.

36. Wu J, Gao W, Tang Q, Yu Y, You W, Wu Z, et al. M2 Macrophage-Derived Exosomes Facilitate HCC Metastasis by Transferring alphaM beta2 Integrin to Tumor Cells. Hepatology. 2021;73(4):1365-80.

37. Park JE, Dutta B, Tse SW, Gupta N, Tan CF, Low JK, et al. Hypoxia-induced tumor exosomes promote M2-like macrophage polarization of infiltrating myeloid cells and microRNA-mediated metabolic shift. Oncogene. 2019;38(26):5158-73.

38. Zhang PF, Gao C, Huang XY, Lu JC, Guo XJ, Shi GM, et al. Cancer cellderived exosomal circUHRF1 induces natural killer cell exhaustion and may cause resistance to anti-PD1 therapy in hepatocellular carcinoma. Mol Cancer. 2020;19(1):110

39. Lu Z, Zuo B, Jing R, Gao X, Rao Q, Liu Z, et al. Dendritic cell-derived exosomes elicit tumor regression in autochthonous hepatocellular carcinoma mouse models. J Hepatol. 2017;67(4):739-48.

40. Xie F, Xu M, Lu J, Mao L, Wang S. The role of exosomal PD-L1 in tumor progression and immunotherapy. Mol Cancer. 2019;18(1):146.

41. Sun C, Mezzadra R, Schumacher TN. Regulation and Function of the PD-L1 Checkpoint. Immunity. 2018;48(3):434-52.

42. Yin Z, Yu M, Ma T, Zhang C, Huang S, Karimzadeh MR, et al. Mechanisms underlying low-clinical responses to PD-1/PD-L1 blocking antibodies in immunotherapy of cancer: a key role of exosomal PD-L1.J Immunother Cancer. 2021;9(1). 
43. Zhang K, Chen J, Li C, Yuan Y, Fang S, Liu W, et al. Exosome-mediated transfer of SNHG7 enhances docetaxel resistance in lung adenocarcinoma. Cancer Lett. 2021.

44. Zheng P, Chen L, Yuan X, Luo Q, Liu Y, Xie G, et al. Exosomal transfer of tumor-associated macrophage-derived miR-21 confers cisplatin resistance in gastric cancer cells. J Exp Clin Cancer Res. 2017;36(1):53.

45. Xin L, Zhou LQ, Liu C, Zeng F, Yuan YW, Zhou Q, et al. Transfer of LncRNA CRNDE in TAM-derived exosomes is linked with cisplatin resistance in gastric cancer. EMBO Rep. 2021:e52124.

46. Binenbaum Y, Fridman E, Yaari Z, Milman N, Schroeder A, Ben David $\mathrm{G}$, et al. Transfer of miRNA in Macrophage-Derived Exosomes Induces Drug Resistance in Pancreatic Adenocarcinoma. Cancer Res. 2018;78(18):5287-99.

47. Zhu X, Shen H, Yin X, Yang M, Wei H, Chen Q, et al. Macrophages derived exosomes deliver miR-223 to epithelial ovarian cancer cells to elicit a chemoresistant phenotype. J Exp Clin Cancer Res. 2019;38(1):81.

48. Zhang H, Deng T, Liu R, Ning T, Yang H, Liu D, et al. CAF secreted miR-522 suppresses ferroptosis and promotes acquired chemo-resistance in gastric cancer. Mol Cancer. 2020;19(1):43.

49. Han M, Hu J, Lu P, Cao H, Yu C, Li X, et al. Exosome-transmitted miR-567 reverses trastuzumab resistance by inhibiting ATG5 in breast cancer. Cell Death Dis. 2020;11(1):43.

50. Salehi M, Sharifi M. Exosomal miRNAs as novel cancer biomarkers: Challenges and opportunities. J Cell Physiol. 2018;233(9):6370-80.

51. Li W, Li C, Zhou T, Liu X, Liu X, Li X, et al. Role of exosomal proteins in cancer diagnosis. Mol Cancer. 2017;16(1):145.

52. Zhou CY, Dong YP, Sun X, Sui X, Zhu H, Zhao YQ, et al. High levels of serum glypican-1 indicate poor prognosis in pancreatic ductal adenocarcinoma. Cancer Med. 2018;7(11):5525-33.

53. Conde-Vancells J, Rodriguez-Suarez E, Gonzalez E, Berisa A, Gil D, Embade $\mathrm{N}$, et al. Candidate biomarkers in exosome-like vesicles purified from rat and mouse urine samples. Proteomics Clin Appl. 2010;4(4):416-25.

54. Kitdumrongthum S, Metheetrairut C, Charoensawan V, Ounjai P, Janpipatkul K, Panvongsa W, et al. Dysregulated microRNA expression profiles in cholangiocarcinoma cell-derived exosomes. Life Sci. 2018;210:65-75.

55. Haga H, Yan IK, Takahashi K, Wood J, Zubair A, Patel T. Tumour cell-derived extracellular vesicles interact with mesenchymal stem cells to modulate the microenvironment and enhance cholangiocarcinoma growth. J Extracell Vesicles. 2015;4:24900.

56. Qin X, Lu M, Li G, Zhou Y, Liu Z. Downregulation of tumor-derived exosomal miR-34c induces cancer-associated fibroblast activation to promote cholangiocarcinoma progress. Cancer Cell Int. 2021;21(1):373.

57. Li L, Piontek K, Ishida M, Fausther M, Dranoff JA, Fu R, et al. Extracellular vesicles carry microRNA-195 to intrahepatic cholangiocarcinoma and improve survival in a rat model. Hepatology. 2017;65(2):501-14.

58. Ota Y, Takahashi K, Otake S, Tamaki Y, Okada M, Aso K, et al. Extracellular vesicle-encapsulated miR-30e suppresses cholangiocarcinoma cell invasion and migration via inhibiting epithelial-mesenchymal transition. Oncotarget. 2018;9(23):16400-17.

59. Wang S, Hu Y, Lv X, Li B, Gu D, Li Y, et al. Circ-0000284 arouses malignant phenotype of cholangiocarcinoma cells and regulates the biological functions of peripheral cells through cellular communication. Clin Sci (Lond). 2019;133(18):1935-53.

60. Xu Y, Leng K, Yao Y, Kang P, Liao G, Han Y, et al. A Circular RNA, Cholangiocarcinoma-Associated Circular RNA 1, Contributes to Cholangiocarcinoma Progression, Induces Angiogenesis, and Disrupts Vascular Endothelial Barriers. Hepatology. 2021;73(4):1419-35.

61. Scavo MP, Depalo N, Rizzi F, Ingrosso C, Fanizza E, Chieti A, et al. FZD10 Carried by Exosomes Sustains Cancer Cell Proliferation. Cells. 2019;8(8).

62. Dutta S, Reamtong O, Panvongsa W, Kitdumrongthum S, Janpipatkul $K$, Sangvanich $P$, et al. Proteomics profiling of cholangiocarcinoma exosomes: A potential role of oncogenic protein transferring in cancer progression. Biochim Biophys Acta. 2015;1852(9):1989-99.

63. Chen JH, Xiang JY, Ding GP, Cao LP. Cholangiocarcinoma-derived exosomes inhibit the antitumor activity of cytokine-induced killer cells by down-regulating the secretion of tumor necrosis factor-alpha and perforin. J Zhejiang Univ Sci B. 2016;17(7):537-44.

64. Lapitz A, Arbelaiz A, O'Rourke CJ, Lavin JL, Casta A, Ibarra C, et al. Patients with Cholangiocarcinoma Present Specific RNA Profiles in Serum and Urine Extracellular Vesicles Mirroring the Tumor Expression: Novel Liquid Biopsy Biomarkers for Disease Diagnosis. Cells. 2020;9(3).
65. Shen L, Chen G, Xia Q, Shao S, Fang H. Exosomal miR-200 family as serum biomarkers for early detection and prognostic prediction of cholangiocarcinoma. Int J Clin Exp Pathol. 2019;12(10):3870-6.

66. Hu C, Zhang Y, Zhang M, Li T, Zheng X, Guo Q, et al. Exosomal Cripto-1 Serves as a Potential Biomarker for Perihilar Cholangiocarcinoma. Front Oncol. 2021;11:730615.

67. Ge X, Wang Y, Nie J, Li Q, Tang L, Deng X, et al. The diagnostic/prognostic potential and molecular functions of long non-coding RNAs in the exosomes derived from the bile of human cholangiocarcinoma. Oncotarget. 2017:8(41):69995-70005

68. Xue XY, Liu YX, Wang C, Gu XJ, Xue ZQ, Zang XL, et al. Identification of exosomal miRNAs as diagnostic biomarkers for cholangiocarcinoma and gallbladder carcinoma. Signal Transduct Target Ther. 2020;5(1):77.

69. Weeraphan C, Phongdara A, Chaiyawat P, Diskul-Na-Ayudthaya P, Chokchaichamnankit D, Verathamjamras C, et al. Phosphoproteome Profiling of Isogenic Cancer Cell-Derived Exosome Reveals HSP90 as a Potential Marker for Human Cholangiocarcinoma. Proteomics. 2019;19(12):e1800159.

70. Ikeda C, Haga H, Makino N, Inuzuka T, Kurimoto A, Ueda T, et al. Utility of Claudin-3 in extracellular vesicles from human bile as biomarkers of cholangiocarcinoma. Sci Rep. 2021;11(1):1195.

71. Arbelaiz A, Azkargorta M, Krawczyk M, Santos-Laso A, Lapitz A, Perugorria MJ, et al. Serum extracellular vesicles contain protein biomarkers for primary sclerosing cholangitis and cholangiocarcinoma. Hepatology. 2017;66(4):1125-43.

72. Valle J, Wasan H, Palmer DH, Cunningham D, Anthoney A, Maraveyas A, et al. Cisplatin plus gemcitabine versus gemcitabine for biliary tract cancer. N Engl J Med. 2010;362(14):1273-81.

73. Shroff RT, Javle MM, Xiao L, Kaseb AO, Varadhachary GR, Wolff RA, et al. Gemcitabine, Cisplatin, and nab-Paclitaxel for the Treatment of Advanced Biliary Tract Cancers: A Phase 2 Clinical Trial. JAMA Oncol. 2019;5(6):824-30

74. Wang J, Ilyas S. Targeting the tumor microenvironment in cholangiocarcinoma: implications for therapy. Expert Opin Investig Drugs. 2021;30(4):429-38.

75. Rizvi S, Khan SA, Hallemeier CL, Kelley RK, Gores GJ. Cholangiocarcinoma - evolving concepts and therapeutic strategies. Nat Rev Clin Oncol. 2018;15(2):95-111.

76. Farshidfar F, Zheng S, Gingras MC, Newton Y, Shih J, Robertson AG, et al. Integrative Genomic Analysis of Cholangiocarcinoma Identifies Distinct IDH-Mutant Molecular Profiles. Cell Rep. 2017;18(11):2780-94.

77. Nakamura H, Arai Y, Totoki Y, Shirota T, Elzawahry A, Kato M, et al. Genomic spectra of biliary tract cancer. Nat Genet. 2015;47(9):1003-10.

78. Tabernero J, Bahleda R, Dienstmann R, Infante JR, Mita A, Italiano A, et al. Phase I Dose-Escalation Study of JNJ-42756493, an Oral Pan-Fibroblast Growth Factor Receptor Inhibitor, in Patients With Advanced Solid Tumors. J Clin Oncol. 2015;33(30):3401-8.

79. Javle M, Roychowdhury S, Kelley RK, Sadeghi S, Macarulla T, Weiss KH, et al. Infigratinib (BGJ398) in previously treated patients with advanced or metastatic cholangiocarcinoma with FGFR2 fusions or rearrangements: mature results from a multicentre, open-label, single-arm, phase 2 study. Lancet Gastroenterol Hepatol. 2021;6(10):803-15.

80. Homet Moreno B, Ribas A. Anti-programmed cell death protein-1/ ligand-1 therapy in different cancers. Br J Cancer. 2015;112(9):1421-7.

81. Piha-Paul SA, Oh DY, Ueno M, Malka D, Chung HC, Nagrial A, et al. Efficacy and safety of pembrolizumab for the treatment of advanced biliary cancer: Results from the KEYNOTE-158 and KEYNOTE-028 studies. Int J Cancer. 2020;147(8):2190-8.

82. Gilligan KE, Dwyer RM. Engineering Exosomes for Cancer Therapy. Int J Mol Sci. 2017;18(6).

83. Bandiera S, Pfeffer S, Baumert TF, Zeisel MB. miR-122-a key factor and therapeutic target in liver disease. J Hepatol. 2015;62(2):448-57.

84. Lou G, Song X, Yang F, Wu S, Wang J, Chen Z, et al. Exosomes derived from miR-122-modified adipose tissue-derived MSCs increase chemosensitivity of hepatocellular carcinoma. J Hematol Oncol. 2015;8:122.

85. Lou G, Chen L, Xia C, Wang W, Qi J, Li A, et al. MiR-199a-modified exosomes from adipose tissue-derived mesenchymal stem cells improve hepatocellular carcinoma chemosensitivity through mTOR pathway. J Exp Clin Cancer Res. 2020;39(1):4.

86. Zhou Y, Zhou W, Chen X, Wang Q, Li C, Chen Q, et al. Bone marrow mesenchymal stem cells-derived exosomes for penetrating 
and targeted chemotherapy of pancreatic cancer. Acta Pharm Sin B. 2020;10(8):1563-75.

87. Chaiyadet S, Sotillo J, Krueajampa W, Thongsen S, Brindley PJ, Sripa B, et al. Vaccination of hamsters with Opisthorchis viverrini extracellular vesicles and vesicle-derived recombinant tetraspanins induces antibodies that block vesicle uptake by cholangiocytes and reduce parasite burden after challenge infection. PLoS Negl Trop Dis. 2019;13(5):e0007450.

88. Gao Y, Zhang H, Zhou N, Xu P, Wang J, Gao Y, et al. Methotrexate-loaded tumour-cell-derived microvesicles can relieve biliary obstruction in patients with extrahepatic cholangiocarcinoma. Nat Biomed Eng. 2020:4(7):743-53

89. Luo C, Xin H, Zhou Z, Hu Z, Sun R, Yao N, et al. Tumor-Derived Exosomes Induce Immunosuppressive Macrophages to Foster Intrahepatic Cholangiocarcinoma Progression. Hepatology. 2022.

90. Chen S, Chen Z, Li Z, Li S, Wen Z, Cao L, et al. Tumor-associated macrophages promote cholangiocarcinoma progression via exosomal Circ_0020256. Cell Death Dis. 2022;13(1):94.

\section{Publisher's Note}

Springer Nature remains neutral with regard to jurisdictional claims in published maps and institutional affiliations.

- fast, convenient online submission

- thorough peer review by experienced researchers in your field

- rapid publication on acceptance

- support for research data, including large and complex data types

- gold Open Access which fosters wider collaboration and increased citations

- maximum visibility for your research: over $100 \mathrm{M}$ website views per year

At BMC, research is always in progress.

Learn more biomedcentral.com/submissions 